\title{
Programa de educação popular em saúde: hábitos de vida e sintomas depressivos em idosos
}

\author{
Popular health education program: lifestyle and depressive symptoms in elderly
}

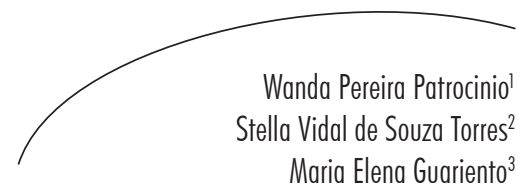

Resumo

Objetivos: Avaliar a proposta de um programa de educação popular em saúde para um envelhecimento saudável em duas comunidades da cidade de Campinas, SP e verificar a influência deste programa sobre os hábitos de vida e em sintomas depressivos dos participantes. Métodos: Trata-se de estudo exploratório, prospectivo e intervencionista. Foram realizadas atividades educacionais com 16 indivíduos idosos, com idades que variavam entre 60 e 75 anos ( $m=66,88 \pm 5$,4anos) e que foram divididos em dois grupos com delineamento pré-teste, intervenção, pós-teste e seguimento; a intervenção consistiu de um encontro semanal de 150 minutos, de agosto a dezembro de 2009. As medidas incluíram questionários e escalas sobre variáveis sociodemográficas, de saúde, hábitos de vida e sintomas depressivos. Os programas foram elaborados com temas escolhidos pelos idosos. Resultados: O estudo mostrou melhora no tempo diário de prática de atividade física; melhora no consumo diário de verduras e legumes para os dois grupos; os participantes de um dos grupos apresentaram piora em sintomas depressivos na medida de seguimento. Conclusões: Os resultados sugerem que intervenções desse tipo podem favorecer a saúde física e mental de idosos, a partir de mudanças de comportamento em saúde; mostra também a importância do grupo social para atenuação dos sintomas depressivos em viúvas.

\section{Abstract}

Objectives: To evaluate the proposal of a program of popular health education to healthy elderly in two communities in the city of Campinas and to assess the influence of this program on the habits of life and depressive symptoms of participants. Methods: This is an exploratory study, prospective and interventional. Educational interventions were performed in 16 elderly subjects with ages ranging from 60 to 75

\footnotetext{
Programa de Pós-graduação em Educação, Faculdade de Educação. Universidade Estadual de Campinas. Campinas, SP, Brasil.

2 Programa de Pós-graduação em Gerontologia, Faculdade de Ciências Médicas. Universidade Estadual de Campinas. Campinas, SP, Brasil.

3 Departamento de Clínica Médica, Faculdade de Ciências Médicas. Universidade Estadual de Campinas. Campinas, SP, Brasil.
}

Palavras-chave: Educação em Saúde. Envelhecimento. Hábitos. Depressão. 
years $(\mathrm{m}=66.88 \pm 5.4$ years $)$ who were divided into two groups with randomized pretest design, intervention, posttest and follow-up, and the intervention consisted of a 150-minute weekly meeting, from August to December 2009. The measures included questionnaires and scales on sociodemographic, health and lifestyle and depressive symptoms. The programs are designed to themes chosen by the elderly. Results: Statistical analysis showed improvement in time daily physical activity, improvement in daily consumption of vegetables for both groups, participants in one group had worsening in depressive symptoms at follow-up measure. Conclusions: The results suggest that such interventions can promote the physical and mental health of older people, reflected in changing health behaviors, also shows the importance of the social group for the reduction and maintenance of depressive symptoms in widows.
Key words: Health

Education. Aging. Habits.

Depression.

\section{INTRODUÇÃ̃o}

O conceito e as práticas modernas de promoção da saúde surgiram e se desenvolveram de forma mais vigorosa nos últimos vinte anos, particularmente no Canadá, nos Estados Unidos e em países da Europa Ocidental. No Brasil, o trabalho voltado para esse tipo de prática entre os usuários de serviços de saúde teve início com as discussões sobre o tema da promoção da saúde (PS), que se manifesta por duas vertentes principais: (1) a educação em saúde realizada de forma mais tradicional nos serviços de atenção básica, representada por palestras e orientações sobre a prevenção de doenças e (2) a que se vincula ao campo da educação popular em saúde. ${ }^{1}$

A promoção em saúde funciona como uma combinação de apoios educacionais e ambientais que visam atingir ações e condições de vida conducentes à saúde. ${ }^{2}$ Para a Organização Mundial da Saúde (OMS), a educação em saúde objetiva desenvolver no indivíduo o senso de responsabilidade por sua própria saúde e pela saúde da comunidade, o que lhe permite ampliar sua capacidade de participar da vida comunitária de maneira construtiva. ${ }^{3}$

No Brasil, com a criação do Sistema Único de Saúde (SUS) nos anos 1980, tornou-se mais evidente a preocupação em concretizar ações populares de saúde. Em 1991, surgiu a proposta de criar uma articulação nacional entre os movimentos de educação popular em saúde, que foi aprofundada em 1996 e, em 2003, deu origem à Articulação Nacional de Movimentos e Práticas de Educação Popular e Saúde (ANEPS). ${ }^{4}$

Em revisão da literatura sobre programas de promoção da saúde do idoso, no período de 1990 a 2002, constatou-se que os objetivos desses programas convergiam para a melhoria da saúde e da qualidade de vida do idoso, com ênfase em mudanças comportamentais e práticas de saúde ou em dimensões subjetivas e sociais do bemestar ou em ambas, combinadas. Os programas pesquisados tinham em comum o fato de recomendarem inovação nas formas de acesso aos programas para que os serviços preventivos baseados em comunidade alcançassem os idosos com maior necessidade. ${ }^{5}$

Foi realizada extensa pesquisa bibliográfica ${ }^{5,6}$ e constatou-se que no Brasil são ainda escassos os estudos sobre educação em saúde orientada a idosos. O primeiro levantamento bibliográfico se deu em 2008 e o segundo em 2010, nas bases de teses e dissertações da Universidade Estadual de Campinas, Universidade de São Paulo, Pontifícia Universidade Católica de São Paulo e Pontifícia Universidade Católica do Rio Grande do Sul, além da SciELO, Ageline e Educational Gerontology, com as palavras-chave: idosos, gerontologia, velhice, envelhecimento cruzando com educação, centros de convivência, educação em saúde, atitudes e sociabilidade, envelhecimento saudável e educação.

A ideia de formar grupos de idosos está se propagando em todas as regiões do país. ${ }^{7}$ Interagir 
socialmente, sobretudo com amigos da mesma geração, possibilita ao idoso construir novos laços de relação e favorece o bem-estar físico, psicológico e social dos idosos. ${ }^{8}$ As deficiências em habilidades sociais parecem constituir um fator de vulnerabilidade para a baixa qualidade de vida e para a depressão em indivíduos da terceira idade..$^{9-10}$

O desenvolvimento de mais pesquisas e de novas práticas sociais pode contribuir para que a sociedade envelheça de maneira mais saudável e positiva. A elaboração e a avaliação de programas educacionais são bons exemplos das possibilidades existentes nessa direção. A pobreza e o baixo nível educacional da maioria dos idosos são condições que só poderão ser superadas em médio e longo prazos. ${ }^{6}$ De imediato, bons programas educacionais oferecidos a idosos podem ajudar a remediar as dificuldades socioeducacionais e culturais desse grupo que cresce rapidamente no país, em comparação com os outros segmentos etários.

Tendo em vista o debate apresentado, o problema desta pesquisa remeteu ao planejamento de programas educacionais dirigidos a idosos. Buscou-se preencher uma lacuna no campo da educação de idosos de modo geral e da educação em saúde em particular, num contexto de escasso reconhecimento dos idosos como uma clientela merecedora de atenção e da existência de estereótipos negativos, segundo os quais os idosos são improdutivos e não flexíveis e, assim, não merecedores de esforços educacionais. ${ }^{6}$

Para a promoção da saúde em idosos, a perspectiva de educação popular ${ }^{11,12}$ parece ser uma ferramenta útil por utilizar conscientização e reflexão e pelo fato de a organização dos conteúdos e das ações ter como ponto de partida os conhecimentos trazidos pelos educandos e a realidade de cada grupo. A proposta de educação desenvolvida por Paulo Freire ${ }^{11-13}$ visa desenvolver nas pessoas o reconhecimento de si mesmas como sujeitos; uma visão crítica sobre as estruturas sociais; e o engajamento ativo em uma prática emancipadora, além de desenvolver processos contínuos e permanentes de formação num contexto de confiança mútua. As atividades devem favorecer a autonomia, a participação, a colaboração, a exploração de pontos de vista alternativos, a problematização e a reflexão crítica. ${ }^{6}$ Além disso, os participantes refletem criticamente sobre os conteúdos e temas e são estimulados a aplicar os novos conhecimentos em sua prática cotidiana. Os participantes são consultados, fazem parte do processo educacional e percebem mais sentido nos conteúdos, o que aumenta a chance de generalização do aprendido para sua vida.

Dessa forma, o objetivo deste estudo foi avaliar a proposta de um programa de educação popular em saúde para um envelhecimento saudável em duas comunidades da cidade de Campinas, SP, e verificar a influência desse programa sobre os hábitos de vida e em sintomas depressivos dos participantes.

\section{METODOLOGIA}

Trata-se de um estudo exploratório, prospectivo e intervencionista, em que foram realizadas intervenções educacionais em dois grupos de idosos residentes em dois bairros do município de Campinas, SP, localizados no Distrito de Saúde Sul, no período de agosto a dezembro de 2009. Entre os cinco distritos desse município, o Sul é o segundo com maior população de idosos (26,8\% do total), segundo dados do Censo atualizados em abril de 2010. ${ }^{14}$ Os idosos estavam referenciados ao Centro de Saúde Orosimbo Maia (grupo 1), que nucleia uma região com índice de vulnerabilidade social (IVS) médio e ao Centro de Saúde Parque da Figueira (grupo 2), que nucleia uma região com IVS baixo.

O recrutamento dos idosos seguiu duas estratégias. Na primeira, os idosos que haviam participado anteriormente de um estudo populacional nacional sobre fragilidade em cada um dos dois bairros selecionados, foram contatados por telefone e pessoalmente pelas mesmas agentes comunitárias de saúde (ACS) que haviam colaborado no recrutamento dos 
participantes daquele estudo. A segunda estratégia consistiu em divulgar o projeto por meio de cartazes e de convites pessoais feitos por uma das pesquisadoras em espaços de participação de idosos. A divulgação foi realizada em igrejas, parques, centros comunitários, centros de convivência e centros de saúde. Os idosos foram convidados a participar de um programa cujo objetivo era proporcionar atividades e informações sobre como ter um envelhecimento saudável. $O$ critério de seleção comunicado aos participantes potenciais era ter 60 anos ou mais de idade e residir na comunidade em que a pesquisa seria desenvolvida.

Os idosos que se apresentaram como voluntários foram orientados a comparecer a uma reunião inicial, na qual foram informados sobre os objetivos do estudo, a duração e o conteúdo da intervenção e os resultados esperados. Compareceram a esse primeiro encontro 26 idosos. Dentre os idosos que estiveram presentes na primeira reunião, 16 aceitaram participar. A eles foram entregues a agenda do programa e as instruções para participação: comparecer para entrevista de realização do pré-teste na data e horário agendados e comparecer aos encontros semanais nos dias e horários acordados pelo grupo. O grupo 1 contou com 11 participantes e o grupo 2, com cinco participantes.

Uma das pesquisadoras realizou avaliações quanto à atividade física, à qualidade da alimentação e à presença de sintomas depressivos. Profissionais das áreas de Fonoaudiologia, Odontologia, Educação Física, Medicina e Psicologia auxiliaram a pesquisadora com conteúdos e testes para o programa. Esses profissionais se reuniram previamente com a pesquisadora para orientação. Foram considerados como ativos os indivíduos que realizavam pelo menos 150 minutos de atividade por semana, distribuídos em cinco dias e em 30 minutos por dia de atividades moderadas, ou que realizavam pelo menos 120 minutos de atividades de forte intensidade, distribuídas em quatro dias e em 30 minutos por dia. ${ }^{15} \mathrm{Em}$ relação à alimentação, seguiram-se as seguintes recomendações: consumo diário de seis porções do grupo dos carboidratos (arroz, pães, massas, batata, mandioca); três porções de frutas; três porções de verduras e legumes e uma porção de leguminosas (feijões) por dia. ${ }^{16}$ Os sintomas depressivos foram avaliados pela aplicação da Escala de Depressão Geriátrica. ${ }^{17,18}$

Foram realizadas medidas de pré-teste, pósteste e seguimento com apoio em instrumentos para avaliação sociodemográfica, psicossocial e de saúde. Tais medidas foram aplicadas individualmente, em situação de entrevista, por uma das pesquisadoras. Em data pré-agendada com os idosos que participaram da reunião de apresentação do projeto, realizou-se a assinatura do Termo de Consentimento Livre e Esclarecido (TCLE) e, na sequência, a aplicação do pré-teste; o pós-teste foi realizado em data combinada na última sessão e o seguimento seis meses depois do término do programa em data e local combinados com os participantes. Os idosos responderam oralmente e as informações foram registradas no protocolo de coleta de dados.

A intervenção consistiu na aplicação de um programa de educação popular em saúde visando o envelhecimento saudável, fundamentado pelo modelo de educação dialógica e popular de Paulo Freire ${ }^{11-13}$ e pela política de envelhecimento ativo da OMS, ${ }^{19}$ cujos pilares são saúde, participação social, proteção e treinamento contínuo. Foi realizado um encontro semanal de cerca de 150 minutos durante quatro meses para cada um dos grupos, totalizando 16 sessões no primeiro grupo e 15 no segundo.

Logo depois do pré-teste, foram apresentados para os participantes os possíveis conteúdos a serem trabalhados na intervenção, pedindo-lhes que apontassem os que lhes pareciam prioritários. Os temas escolhidos pelos grupos foram: memória; distúrbios do sono; atividade física; alimentação saudável; saúde bucal; emoções e 
sentimentos na velhice; uso de medicamentos; instruções sobre saúde; imagem positiva do envelhecimento; maus-tratos a idosos e atividades terapêuticas (lian gong, tai chi chuan, yoga, massagem e argila terapêutica). Os programas foram organizados com base nesses temas.

No início de cada sessão, a pesquisadora apresentava o tema para os participantes e provocava uma discussão ou reflexão sobre o assunto, estimulando a participação ora do grupo, ora do indivíduo, e a partir disso a aula se desenvolvia. Foram adotadas várias formas de desenvolvimento dos encontros educativos: dinâmicas, bate-papo, atividades corporais, leitura de textos, exercícios práticos e oficinas.

Para comparação das variáveis categóricas, foi utilizado o teste qui-quadrado ou o teste exato de Fisher. Para comparar as variáveis numéricas entre dois grupos, foi utilizado o teste de Mann-Whitney. Para comparar as variáveis categóricas dicotômicas entre as três avaliações (pré-teste, pós-teste e seguimento), foi utilizado o teste de Cochran; e para comparar as variáveis categóricas ordinais entre as três avaliações, foi utilizado o teste de Friedman. Para comparar as variáveis numéricas entre as três avaliações e os dois grupos, foi utilizada análise de variância para medidas repetidas (ANOVA com medidas repetidas). O nível de significância adotado para os testes estatísticos foi de $5 \%$.

$\mathrm{Na}$ ocasião da entrevista individual, os idosos assinaram o TCLE, que integra o projeto aprovado pelo Comitê de Ética em Pesquisa da FCM/Unicamp sob o número 449/2009.

\section{RESULTADOS}

A idade média do grupo foi de 66,88 anos ( $\pm 5,4$ anos, mínima 60 e máxima 75 anos), sendo a maioria na faixa etária de 60 a 69 anos. Eram 15 mulheres e um homem. Nove participantes eram aposentados e cinco recebiam pensão. A escolaridade de 11 participantes correspondia ao primeiro ciclo do ensino fundamental e 13 pessoas tinham uma renda mensal individual de até três salários mínimos. Todas as participantes vinculadas à Unidade do Parque da Figueira eram viúvas e seis dos participantes da outra unidade eram casados. Em relação à experiência profissional, sete pessoas tinham atuado como faxineiras, empregadas domésticas e vendedoras; cinco eram donas de casa; uma era operadora de máquinas e um forneiro industrial; uma era professora e outra auxiliar de enfermagem.

A doença crônica mais comum era hipertensão arterial e o problema de saúde mais comum era medo de cair. Além disso, todos os idosos faziam uso de medicamentos, sendo a média de 2,82 remédios por dia. Uma pessoa fumava e nenhuma fazia uso excessivo de bebida alcoólica.

Considerando-se a atividade física dos 16 idosos do estudo, nas três medidas (pré-teste, pós-teste e seguimento), metade dos idosos era ativa e metade sedentária. Apenas um idoso mudou de categoria do pré-teste para o pós-teste, passando de sedentário a ativo. Houve diferença estatisticamente significativa $(\mathrm{p}=0,046)$ no tempo diário de prática de atividade física entre o pré-teste e o pós-teste no primeiro grupo, conforme indica a tabela 1. 
Tabela 1- Tempo diário de atividade física. Campinas, SP, 2009.

\begin{tabular}{|c|c|c|c|c|}
\hline & \multicolumn{4}{|c|}{ Grupo 1} \\
\hline Variável & $\mathrm{n}$ & Média & $\mathrm{dp}$ & Mediana \\
\hline $\begin{array}{l}\text { Tempo diário (min.) } \\
\text { (pré-teste) }\end{array}$ & 9 & 57,78 & 16,41 & 60,00 \\
\hline \multirow{2}{*}{$\begin{array}{l}\text { Tempo diário (min.) } \\
\text { (pós-teste) }\end{array}$} & 10 & 60,00 & 26,56 & 55,00 \\
\hline & \multicolumn{4}{|c|}{ Grupo 2} \\
\hline $\begin{array}{l}\text { Tempo diário (min.) } \\
\text { (pré-teste) }\end{array}$ & 5 & 46,00 & 34,53 & 40,00 \\
\hline \multirow[t]{2}{*}{$\begin{array}{l}\text { Tempo diário (min.) } \\
\text { (pós-teste) }\end{array}$} & 5 & 37,00 & 12,55 & 40,00 \\
\hline & \multicolumn{4}{|c|}{$\begin{array}{l}\text { Valor- } \mathrm{p} \text { referente ao teste de Mann-Whitney para comparação dos valore } \\
\text { entre os dois grupos }\end{array}$} \\
\hline Variável & \multicolumn{4}{|c|}{ Valor-p } \\
\hline $\begin{array}{l}\text { Tempo diário (min.) } \\
\text { (pré-teste) }\end{array}$ & \multicolumn{4}{|c|}{$\mathrm{p}=0,163$} \\
\hline $\begin{array}{l}\text { Tempo diário (min.) } \\
\text { (pós-teste) }\end{array}$ & \multicolumn{4}{|c|}{$\mathrm{p}=0,046$} \\
\hline
\end{tabular}

As atividades relatadas pelos idosos dos dois grupos foram: ginástica (sete participantes), alongamentos (três), caminhada (sete), lian gong (cinco), tai chi chuan (um), hidroginástica (um) e yoga (um) no pré-teste; ginástica (quatro), alongamentos (quatro), caminhada (sete), lian gong (sete), tai chi chuan (um), hidroginástica (um) e yoga (um) no pós-teste; ginástica (quatro participantes), alongamentos (um), caminhada (10), lian gong (sete), tai chi chuan (um), hidroginástica (zero) e yoga (um) no seguimento.

Em relação à alimentação, no pré-teste registrou-se uma média de consumo de 2,56 frutas por dia; consumo de legumes e verduras em média 1,81 vezes por dia; carboidratos 2,81 vezes por dia e de feijões e leguminosas 1,53 vezes por dia. No pós-teste, houve aumento no consumo em todos os itens, passando para a média de três frutas; 1,88 vezes de legumes e verduras; 3,06 vezes de carboidratos e 1,60 vezes de feijões e leguminosas. Na medida de seguimento, manteve-se a melhora na média do consumo de verduras e legumes e de carboidratos (médias de 2,88 frutas; 2,06 vezes de legumes e verduras; 3,63 vezes de carboidratos e 1,47 vezes de feijões). Houve diferença significativa $(\mathrm{p}=0,030)$ quanto ao consumo diário de verduras e legumes no pré-teste para o pós-teste para o grupo 1, e diferença significativa $(\mathrm{p}=0,014)$ entre o pós-teste e o seguimento para o grupo 2, conforme indica a figura 1 . 
Consumo diário de verduras e legumes

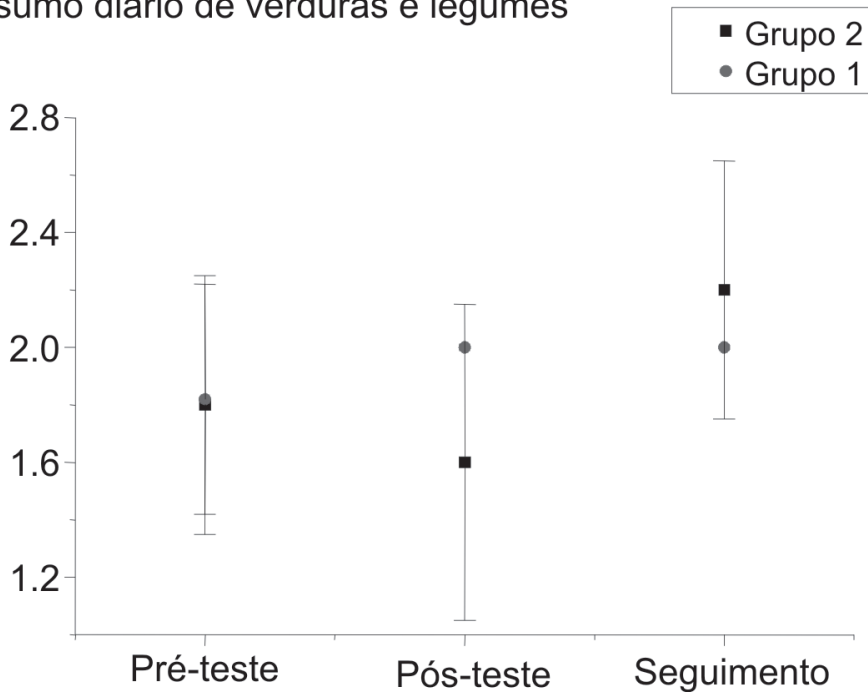

Figura 1 - Distribuição das porções de consumo diário de verduras e legumes. Campinas, SP, 2009.

No tocante aos sintomas depressivos, no pré-teste, três participantes do segundo grupo e apenas um do primeiro grupo pontuaram para essa variável. No pós-teste, nenhum do grupo 1 e dois participantes do grupo 2 pontuaram para essa variável. No seguimento, foram três participantes com sintomas depressivos no segundo grupo e nenhum no grupo 1. Registrouse diferença significativa quanto à presença de sintomas depressivos no segundo grupo quando se comparou o seguimento com o pós-teste $(\mathrm{p}=0,018)$.

Tabela 2 - Distribuições de frequência dos idosos com e sem sintomas depressivos. Campinas, SP, 2009.

\begin{tabular}{|c|c|c|c|c|}
\hline \multirow[b]{2}{*}{ Variável } & \multicolumn{2}{|c|}{ Grupo 1} & \multicolumn{2}{|c|}{ Grupo 2} \\
\hline & Sim & Não & Sim & Não \\
\hline $\begin{array}{l}\text { Sintomas depressivos } \\
\text { (pré-teste) }\end{array}$ & $9,09 \%$ & $90,91 \%$ & $60,00 \%$ & $40,00 \%$ \\
\hline $\begin{array}{l}\text { Sintomas depressivos } \\
\text { (pós-teste) }\end{array}$ & $0,00 \%$ & $100 \%$ & $40,0 \%$ & $60,00 \%$ \\
\hline $\begin{array}{l}\text { Sintomas depressivos } \\
\text { (seguimento) }\end{array}$ & $0,00 \%$ & $100 \%$ & $60,00 \%$ & $40,00 \%$ \\
\hline
\end{tabular}




\section{DISCUSSÃO}

O presente estudo buscou avaliar o impacto de um programa de educação popular em saúde e envelhecimento saudável sobre hábitos de vida e sintomas depressivos aplicado a idosos comunitários. Há escassez de trabalhos avaliativos sobre as atividades realizadas com grupos de idosos, ${ }^{6,20}$ vários estudos são relatos de experiências com aspectos avaliativos de base qualitativa e não propriamente pesquisas com fins de avaliação. ${ }^{5}$

Em relação aos grupos de promoção da saúde (GPS), ${ }^{21}$ houve uma ampliação em dois sentidos: primeiro, o GPS é uma intervenção coletiva interdisciplinar de saúde e o presente estudo buscou ir além da saúde, trabalhando juntamente outros temas orientados pela política de envelhecimento ativo da OMS ${ }^{19}$ segundo, ao realizar o programa em espaços comunitários houve um deslocamento de ambientes de tratamento de doenças e maior investimento em atividades de autocuidado e prevenção. Além disso, buscou-se contribuir com as pesquisas na área de educação em saúde realizadas na comunidade, já que na revisão da literatura realizada $^{22}$ as práticas educativas em saúde aconteceram com maior frequência na atenção terciária (unidade hospitalar) e não na atenção primária (unidades de atenção básica na área da saúde da família, centros de saúde e comunidade).

$\mathrm{Na}$ questão da saúde, acreditava-se que o programa poderia influenciar nos hábitos de vida indicados por atividade física e qualidade de alimentação. $\mathrm{Na}$ atividade física, esta pesquisa encontrou $50 \%$ de idosos ativos e $50 \%$ de sedentários, resultado próximo ao de outro estudo ${ }^{23}$ na comunidade, em que foram encontrados $58 \%$ de idosos ativos. Na pesquisa SESC/FPA ${ }^{24}$ (Serviço Social do Comércio/Fundação Perseu Abramo), foram relatados 57\% de idosos (60-64 anos) que realizavam atividade física regularmente e, em outro estudo, ${ }^{20} 60 \%$ de idosos.

Com base nos resultados do programa de educação popular em saúde, não houve influência significativa na mudança de comportamento dos participantes quanto à prática de atividade física. Houve, porém, aumento significativo no tempo de atividade semanal para o primeiro grupo, o que pode ter sido influenciado pelas orientações do programa nas discussões sobre a importância da prática de atividade física para manutenção da saúde e prevenção de doenças e as correções individuais na planilha de atividade física de cada indivíduo. Ao longo do desenvolvimento deste tema, os idosos preencheram uma planilha informando que atividades desenvolviam ao longo da semana e qual o tempo de duração de cada uma delas. Após as discussões sobre o assunto, eles foram orientados sobre como poderiam melhorar sua prática de acordo com as recomendações dirigidas a idosos. ${ }^{17} \mathrm{Um}$ dos idosos sedentários passou a realizar caminhada durante a realização do programa, atividade que foi mantida após o término. As atividades de ginástica, alongamento, caminhada e lian gong realizadas pelos idosos foram promovidas pelo município, exceto hidroginástica e yoga, que alguns idosos praticavam em locais privados.

Quanto à ingestão de carboidratos, no pré-teste os idosos consumiam abaixo do recomendado, porém no pós-teste e seguimento houve aumento da quantidade ingerida, embora continuassem a não consumir os valores recomendados; no consumo de frutas, estavam um pouco abaixo do recomendado, passaram ao consumo ideal no pós-teste e diminuíram um pouco no seguimento; nas verduras e legumes, registrou-se $\mathrm{o}$ resultado mais significativo quanto ao aumento do consumo no pós-teste e no seguimento, porém ainda não foram atingidas as recomendações mínimas. O único critério em que os idosos atenderam às recomendações foi no consumo de feijões e leguminosas.

Em estudo realizado no município de São Paulo, ${ }^{25}$ um quinto dos participantes consumia diariamente as porções recomendadas pela OMS $^{26}$ (consumo de cinco ou mais porções de frutas e hortaliças), enquanto outros $35 \%$ não consumiam diariamente nenhum tipo de fruta ou hortaliça, sendo que esse consumo aumentava significativamente conforme $\mathrm{O}$ 
nível de escolaridade e a renda per capita dos indivíduos. Em função das mudanças fisiológicas relacionadas ao envelhecimento, bem como da maior prevalência de doenças crônicas, estudos e recomendações mostram que uma alimentação adequada é uma das estratégias mais eficazes para a promoção da saúde, e que o consumo habitual de alimentos que contenham nutrientes essenciais reduz o risco de doenças cardiovasculares, neoplasias e distúrbios nutricionais, como obesidade e desnutrição. ${ }^{27}$

Além disso, o presente estudo mostrou que é relativamente difícil alterar hábitos alimentares, porque respondem a fortes determinações culturais e socioeconômicas. $\mathrm{Na}$ velhice, a mudança é mais difícil por causa da presença concomitante de doenças crônicas e problemas de saúde. Para os diabéticos, hipertensos e portadores de dislipidemia, o consumo de grãos integrais é importante, pois a presença das fibras teria papel na saciedade, devido a sua maior viscosidade, que promove um atraso no esvaziamento gástrico, na absorção intestinal ou em ambos. Alguns estudos mostram que os grãos proporcionam um efeito baixo e lento na concentração de glicose no sangue, com menores índices de resistência à insulina. ${ }^{28}$

No tocante aos sintomas depressivos, $60 \%$ das idosas do grupo 2 apresentaram sintomas depressivos na medida de seguimento em comparação ao pós-teste, embora o grupo tenha apresentado melhora entre o pré-teste e o pósteste. Acredita-se que esse achado se associe a uma característica específica das mulheres desse grupo, a viuvez. Em estudo ${ }^{29}$ realizado com participantes idosos viúvos que estavam em processo de luto nos últimos 12 meses, os autores encontraram relação com maior presença de sintomas depressivos quando comparados aos casados. Mesmo que nogrupo pesquisado as idosas já fossem viúvas há mais tempo, nos encontros relataram solidão e encontraram no grupo uma forma de socialização, sentindo-se confortadas. De acordo com pesquisa ${ }^{10}$ realizada com idosos frequentadores de um centro de convivência em comparação com idosos não frequentadores, há menor prevalência de depressão nos idosos que frequentam esses grupos, pois quanto mais amigos e mais atividades sociais, menor a sintomatologia depressiva.

Durante as aulas, houve maior interesse por temas como problemas de saúde e doenças, alimentação, memória e práticas de autocuidado, que são recorrentes na velhice..$^{30} \mathrm{Na}$ literatura, verifica-se que as doenças e condições mórbidas mais prevalentes entre os idosos são: hipertensão arterial $\left(53,4 \%\right.$ no $\mathrm{SABE}^{31}$ (Saúde, Bem-estar e Envelhecimento), 43\% no SESC/FPA ${ }^{24}$ e 68,76\% nesta pesquisa); problemas osteoarticulares (33,8\% de artropatias no SABE, ${ }^{29} 29 \%$ no SESC/ FPA, ${ }^{24} 43,75 \%$ de osteoporose e $37,5 \%$ de artrite ou reumatismo na presente pesquisa) e problemas cardíacos (20,6\% no SABE, ${ }^{31} 13 \%$ no SESC/ FPA $^{24}$ e $37,5 \%$ nesta pesquisa). Com a elevada prevalência dessas enfermidades em idosos, era esperado que os participantes tivessem interesse em discutir estas questões.

No primeiro grupo, aqueles que tinham alguma das doenças anteriormente citadas puderam compartilhar informações sobre como lidavam com ela no cotidiano. No segundo grupo, as idosas que relataram diabetes e osteoporose compartilharam informações com outras que recentemente tinham recebido diagnóstico dessas doenças, que, por sua vez, manifestaram sua experiência quanto à prática dessas sugestões. $\mathrm{Na}$ medida de seguimento, os participantes relataram que continuavam a realizar as práticas de prevenção de doenças, que envolviam o cuidado com a alimentação, a atividade física, o estilo de vida e a prevenção de complicações das doenças já diagnosticadas.

Quanto à alimentação, já é do conhecimento popular que, quando balanceada, constitui um dos melhores meios de promover a saúde, e que dela depende o controle adequado de algumas enfermidades, como diabetes e hipertensão arterial. Os idosos desta pesquisa foram recrutados, em grande parte, pelas ACS, nos serviços oferecidos em dois centros de saúde do município de Campinas, SP. Nessas unidades, em datas pré-agendadas, ocorrem encontros de 
aconselhamento e orientação sobre alimentação como prática preventiva de doenças. Por isso, acredita-se que houve grande interesse sobre o tema. Os idosos puderam trocar receitas culinárias e esclareceram dúvidas.

Houve resultado significativo em relação ao aumento do consumo de verduras e legumes na dieta. Em contrapartida, não foram observadas mudanças quantitativas no consumo dos outros alimentos, possivelmente em decorrência de hábitos culturais e de fatores econômicos. Em estudo $^{32}$ realizado em um centro de convivência no município de São Paulo, observou-se a influência dos fatores econômicos no comportamento alimentardos idosos. No pós-teste, os participantes disseram ter havido aumento de conhecimento sobre alimentação saudável e sobre mudanças práticas na nutrição. Esses relatos reapareceram no seguimento, em que vários idosos alegaram mudança no modo de se alimentar.

Em relação às práticas de autocuidado, a escolha de atividades se deu com base na experiência de uma das pesquisadoras com grupos de adultos e de idosos e nas práticas vigentes no SUS, por meio da Política Nacional de Práticas Integrativas e Complementares. ${ }^{33} \mathrm{Em}$ avaliação realizada em 2006, ${ }^{33}$ as práticas mais desenvolvidas no território brasileiro eram reiki, lian gong, tai chi chuan, automassagem, shiatsu, yoga, shantala, tuiná e lien chi. Na presente pesquisa, utilizaram-se as atividades de automassagem, lian gong, tai chi chuan, yoga, ritos tibetanos e argila medicinal. Foi realizada uma pesquisa ${ }^{34}$ em três municípios (dois no Rio de Janeiro e um em Minas Gerais) que desenvolviam programas ou serviços públicos que contemplavam o exercício de práticas voltadas para a integralidade da atenção e do cuidado em saúde. Em um dos municípios do Rio de Janeiro foram oferecidas práticas de yoga, sendo que os médicos constataram significativa melhora no quadro clínico dos pacientes acometidos por diabetes e hipertensão arterial, observando-se a redução dos níveis pressóricos e de glicemia.
Em outro estudo, ${ }^{35}$ também no Rio de Janeiro, a prática do tai chi cbuan propiciou um conhecimento maior dos indivíduos a respeito de si mesmos, de seu corpo e de seu psiquismo, com a consequente busca de maior autonomia diante do processo de adoecimento, facilitando um projeto de reconstrução da própria saúde, pela qual os pacientes passaram a ser menos dependentes de medicamentos e de médicos. No pós-teste da presente pesquisa, os idosos relataram a importância do aprendizado de exercícios e de práticas de autocuidado no cotidiano. Na medida de seguimento, relataram que continuavam a praticar as atividades aprendidas e a cuidar melhor de si.

O desenvolvimento de atividades que vão além do tema "doenças" proporcionou alterações nos hábitos de vida dos participantes. Semelhante percepção foi encontrada em estudo $^{36}$ realizado com idosos que frequentam uma UnATI (Universidade Aberta da Terceira Idade) na cidade de São Paulo, em que os autores afirmam que não se trata apenas de proporcionar informações que os ajudem a combater os efeitos do envelhecimento; é vital que sejam oferecidos conhecimentos e atividades que favoreçam a criatividade, expressividade, participação e, principalmente, atividades que incrementem sua qualidade de vida.

Uma limitação deste estudo foi o número de participantes nos dois grupos, em decorrência da dificuldade de adesão, que restringe a generalização. Entretanto, naqueles que foram avaliados, podem-se constatar os efeitos benéficos da intervenção educacional na área da saúde. Sugere-se, para pesquisas futuras, a replicação da metodologia utilizada junto a um maior número de grupos de idosos, provenientes de comunidades com diferentes perfis socioeconômicos. Além disso, pode ser interessante realizar esse programa com adultos e acompanhá-los ao longo do tempo para verificar a eficácia no processo de envelhecimento dessas pessoas. 


\section{CONCLUSÕES}

Para a área da educação em saúde, o programa pode contribuir para os serviços existentes nos centros de saúde, como, por exemplo, os Grupos de Qualidade de Vida. Esses grupos podem se beneficiar da metodologia participativa, em que os profissionais realizem um levantamento de interesses dos participantes, contribuindo para que mudem seus comportamentos em saúde.

A implantação de um programa de educação popular em saúde visando à satisfação das necessidades psicológicas e de saúde de idosos residentes na comunidade resultou em subsídios para o desenvolvimento de métodos práticos. Com base no estudo ora relatado, outros programas de intervenção podem ser propostos para os vários níveis de atenção à educação e à saúde da população idosa.

Em termos dos conteúdos desenvolvidos, os programas futuros poderão usar métodos de avaliação mais refinados que permitam averiguar a variação no número de medicamentos, no desempenho em memória e nas práticas de autocuidado.

\section{REFERÊNCIAS}

1. Buss PM, Carvalho AI. Desenvolvimento da promoção da saúde no Brasil nos últimos vinte anos (1988-2008). Ciênc Saúde Coletiva 2009;14(6):2305-16.

2. Candeias NMF. Conceitos de educação e de promoção em saúde: mudanças individuais e mudanças organizacionais. Rev Saúde Pública 1997;31(2):209-13.

3. Levy SN, Silva JJC, Cardoso IFR, Werberich PM, Moreira LLS, Montiani H, et al. Educação em saúde: histórico, conceitos e propostas. Brasília: Ministério da Saúde; 1997 [acesso em 5 jan 2010]. 22 f. Disponível em: www.datasus.gov.br/cns/temas/ educacaosaude/educacaosaude.htm.

4. Severo DO, Cunha AP, Ros MA. Articulação nacional de movimentos e práticas de educação popular e saúde no estado de Santa Catarina: fortalezas e fragilidades. Texto Contexto Enferm 2007;16(2):239-45.

5. Assis M, Hartz ZMA, Valla VV. Programas de promoção da saúde do idoso: uma revisão da literatura científica no período de 1990 a 2002. Ciênc Saúde Coletiva 2004;9(3):557-81.

6. Patrocinio WP. Descrição e análise dos efeitos de um programa de educação popular em saúde dirigido a idosos comunitários [tese de Doutorado]. Campinas: Faculdade de Educação da UNICAMP; 2011.

7. CNBB. Vida, dignidade e esperança. Fraternidade e pessoas idosas. Campanha da Fraternidade. Texto Base CF 2003. São Paulo: Editora Salesiana; 2002.

8. Neri AL, Sommerhalder C. As várias faces do cuidado e do bem-estar do cuidador. In: Neri AL, organizadora. Cuidar de idosos no contexto da família: questões psicológicas e sociais. Campinas: Alínea; 2001. p. 9-62.

9. Carneiro RS, Falcone E, Clark C, Del Prette Z, Del Prette A. Qualidade de vida, apoio social e depressão em idosos: relação com habilidades sociais. Psicol Reflex Crít 2007,20(2):229-37.

10. Almeida EA, Madeira GD, Arantes, PMM, Alencar MA. Comparação da qualidade de vida entre idosos que participam e idosos que não participam de grupos de convivência na cidade de Itabira-MG. Rev Bras Geriatr Gerontol 2010;13(3):435-44.

11. Freire P. Pedagogia do oprimido. 11. ed. Rio de Janeiro: Paz e Terra; 1982. 218 p

12. Freire P. Educação e mudança. 24. ed. Rio de Janeiro: Paz e Terra; 1979. 79 p.

13. Freire P. Extensão ou comunicação? 2. ed. Rio de Janeiro: Paz e Terra; 1975. 136 p.

14. Prefeitura Municipal de Campinas. Sistemas de Informações em Saúde - Tabnet. Campinas: Secretaria de Saúde; 2010 [acesso em 6 jan 2010]. Disponível em: http://2009.campinas.sp.gov.br/saude/tabnet-home/ index.htm.

15. Nelson ME, Rejeski WJ, Blair SN, Duncan PW, Judge JO, King AC, et al. Physical activity and public health in older adults: recommendation from the american college of sports medicine and the american heart association. Med Sci Sports Exerc 2007;39(8):1435-45. 
16. Brasil. Ministério da Saúde. Secretaria de Atenção à Saúde. Coordenação-Geral da Política de Alimentação e Nutrição. Guia alimentar para a população brasileira: promovendo a alimentação saudável. Série A. Normas e manuais Técnicos. Brasília: Ministério da Saúde; 2006. 210 p. [acesso em 10 ago 2009]. Disponível em: http://189.28.128.100/nutricao/docs/ geral/guia_alimentar_conteudo.pdf

17. Yesavage JA, Brink TL, Rose TL, Lum O, Huang $\mathrm{V}$, Adey M, et al. Development and validation of a geriatric depression screening scale: a preliminary report. J Psychiatr Res 1982-1983;17(1):37-49.

18. Almeida OP, Almeida SA. Short versions of the Geriatric Depression Scale: A study of their validity for the diagnosis of major depressive episode according to ICD-10 and DSM-IV. Inter J Geriatr Psychiatry 1999;14(10):858-65.

19. Organização Mundial da Saúde. Envelhecimento ativo: uma política de saúde. Brasília: Organização Pan-Americana da Saúde; 2005. 60 p

20. Assis M, Hartz ZMA, Pacheco LC, Valla VV. Avaliação do projeto de promoção da saúde do Núcleo de Atenção ao Idoso: um estudo exploratório. Interface Comun Saúde Educ 2009;13(29):367-82.

21. Santos LM, Da Ros MA, Crepaldi MA, Ramos LR. Grupos de promoção à saúde no desenvolvimento da autonomia, condições de vida e saúde. Rev Saúde Pública 2006;40(2):346-52.

22. Carvalho VLS, Clementino VQ, Pinho LMO. Educação em saúde nas páginas da REBEn no período de 1995 a 2005. Rev Bras Enferm 2008;61(2):243-8.

23. Siqueira FV, Nahas MV, Facchini LA, Silveira DS, Piccini RX, Tomasi E, et al. Aconselhamento para a prática de atividade física como estratégia de educação à saúde. Cad Saúde Pública 2009;25(1):203-13.

24. Lebrão ML, Duarte YAO. Saúde e independência: aspirações centrais para os idosos. Como estão sendo satisfeitas? In: Neri AL, organizadora. Idosos no Brasil: vivências, desafios e expectativas na terceira idade. São Paulo: Editora Fundação Perseu Abramo, Edições SESC; 2007. p. 191-207.

25. Viebig RF, Pastor-Valero M, Scazufca M, Menezes PR. Consumo de frutas e hortaliças por idosos de baixa renda na cidade de São Paulo. Rev Saúde Pública 2009;43(5):806-13.

26. World Health Organization. Global Strategy on diet, physical activity and health. Fifty seventy word health assembly. Geneva: WHO; 2004 [acesso em 15 fev 2010]. Disponível em: http://www.who.int/ dietphysicalactivity/en/.

27. White HJ. Condições funcionais para alimentação, estado nutricional, perda de peso e autoavaliação de saúde em idosos comunitários: dados do FIBRA Campinas [dissertação de Mestrado]. Campinas: Programa de Pós-Graduação em Gerontologia da Faculdade de Ciências Médicas da UNICAMP; 2010.

28. Mello VD, Laaksonen DE. Fibras na dieta: tendências atuais e benefícios à saúde na síndrome metabólica e no diabetes melito tipo 2. Arq Bras Endocrinol Metab 2009;53(5):509-518.

29. Trentini CM, Werlang BSG, Xavier FMF, Argimon IIL. A relação entre variáveis de saúde mental e cognição em idosos viúvos. Psicol Reflex Crít 2009;22(2):236-43.

30. Neri AL. Qualidade de vida na velhice: enfoque multidisciplinar. 2. ed. Campinas: Alínea; 2011. 308 p.

31. Alves LC, Leimann BCQ, Vasconcelos MEL, Carvalho MS, Vasconcelos AGG, Fonseca TCO, et. al. A influência das doenças crônicas na capacidade funcional dos idosos do Município de São Paulo, Brasil. Cad Saúde Pública 2007;23(8):1924-30.

32. Santos GD, Ribeiro SML. Aspectos afetivos relacionados ao comportamento alimentar dos idosos frequentadores de um Centro de Convivência. Rev Bras Geriatr Gerontol 2011;14(2):319-28.

33. Brasil. Ministério da Saúde. Secretaria de Atenção à Saúde. Departamento de Atenção Básica. Política Nacional de Práticas Integrativas e Complementares no SUS: atitude de apmliação de acesso. Série B. Textos Básicos de Saúde. Brasília: Ministério da Saúde; 2006. 92 p.

34. Pinheiro R, Guizardi FL. Cuidado e integralidade: por uma genealogia de saberes e práticas no cotidiano. In: Pinheiro R. e Mattos RA. Cuidado: as fronteiras da integralidade. 4. ed. Rio de Janeiro: CEPESC/ IMS/UERJ/ABRASCO; 2008.

35. Luz MT. Novos saberes e práticas em saúde coletiva: estudos sobre racionalidades médicas e atividades corporais. 3. ed. São Paulo: Hucitec; 2007. 174 p.

36. Ordonez TN, Cachioni M. Motivos para frequentar um programa de educação permanente: relato dos alunos da universidade aberta à terceira idade da Escola de Artes, Ciências e Humanidades da Universidade de São Paulo. Rev Bras Geriatr Gerontol 2011;14(3):461-74. 